\title{
Henry Bitterfeld, Thomas Aquinas and Identifying Sources in Late Medieval Texts. A Case Study of De formatione et reformatione Ordinis Fratrum Praedicatorum*
}

\begin{abstract}
Zarys treści: Artykuł poświęcony jest sposobom wykorzystania tekstów św. Tomasza z Akwinu w napisanym u schyłku XIV w. traktacie Henryka Bitterfelda z Brzegu De formatione et reformatione Ordinis Fratrum Praedicatorum. W dziele Bitterfelda Akwinata jest nie tylko najczęściej cytowanym autorytetem, ale jego teksty stanowią dla autora budulec dla jego własnego tekstu. Szczególne miejsce dzieła Akwinaty w traktacie śląskiego dominikanina wynika z praktycznej realizacji założeń dominikańskiego ruchu obserwanckiego, na potrzeby którego powstał analizowany traktat, wzywający do wierności zakonnym przepisom. Te nakazywały wszystkim dominikanom studiowanie i propagowanie nauczania św. Tomasza.
\end{abstract}

\begin{abstract}
The article deals with the ways in which St Thomas Aquinas's texts were used by Henry Bitterfeld in his late-14 ${ }^{\text {th }}$-century treatise De formatione et reformatione Ordinis Fratrum Praedicatorum. Not only is Aquinas the most often quoted authority in Bitterfeld's writing but also his texts are the building materials for Bitterfeld. This special place of St Thomas in the treatise by the Silesian Dominican resulted from a practical realisation of the assumptions of the Dominican Observant Reform for the needs of which the analysed treatise was written, calling for the return of the Dominicans to faithful observance of the Order's regulations. Those ordered the friars to study and propagate St Thomas's teachings.
\end{abstract}

Słowa kluczowe: dominikanie, średniowiecze, Henryk Bitterfeld z Brzegu, reforma Kościoła, edycja źródeł średniowiecznych

Keywords: Order of Preachers, Middle Ages, Henry Bitterfeld of Brzeg, Church Reform, editing medieval texts

De formatione et reformatione Ordinis Fratrum Praedicatorum ${ }^{1}$ by Henry Bitterfeld of Brzeg $^{2}$ was the first work of its type written for the needs of the Observant Reform of the Dominican Order that

\footnotetext{
* The text has been written as part of grant no. UMO-2018/31/B/HS3/01196 "Henry of Bitterfeld and the Observant Reform of the Order of Preachers. Critical edition of the De formatione et reformatione Ordinis Fratrum Praedicatorum treatise" financed by the National Science Centre, Poland.

1 The most recent studies of the text can be found in two articles: A. Zajchowska, Formatio - deformatio - reformatio. O reformie obserwanckiej Zakonu Braci Kaznodziejów w świetle traktatu "De (de)formatione et reformatione" Henryka Bitterfelda, in: "Ecclesia semper reformanda". Kryzysy i reformy średniowiecznego Kościoła, ed. T. Gałuszka, T. Graff, G. Ryś, Kraków 2013, p. 507-514; ead., “Quomodo cecidisti?” - kryzys w Zakonie Braci Kaznodziejów w średniowieczu w świetle traktatu Henryka Bitterfelda „De formatione et reformatione Ordinis Fratrum Praedicatorum”, “Czas Przeszły. Poznańskie Studia Historyczne", 4, 2017, p. 125-135. A critical edition of the text will be published in 2022.

2 The most comprehensive and still valid article on Bitterfeld is by V.J. Koudelka, Heinrich Bitterfeld. Profesor an der Universität Prag, "Archivum Fratrum Praedicatorum", 23, 1953, p. 5-65. Also, Magdalena Mill-Papuzińska summarises
} 
began at the end of the $14^{\text {th }}$ century. ${ }^{3}$ It was probably composed at the request of the General Master of the Order, Raymond of Capua, who officially introduced the reform in $1390 .{ }^{4}$ The main goal of the reform was the return of the Dominicans to the faithful observance of the Order's law. ${ }^{5}$ In 1309, the General Chapter of the Order required all Dominican teachers "quod legant et determinent secundum doctrinam et opera venerabilis doctoris fratris Thome de Aquino". ${ }^{6}$ This decision was repeated many times. According to this law, the friars were obliged to study and propagate his writings. All attempts to withdraw from this rule were seen as offences against the unity of the Order. ${ }^{7}$ It is, therefore, worth asking what the place of Aquinas's teaching in the work of Bitterfeld was. Did he, as an observant friar eager to fulfil all the requirements of the law, know Aquinas's writings and was he ready to base his teaching on Aquinas's doctrine?

To answer these questions, we have to analyse the sources of Bitterfeld's text. As with all the other works of this author, ${ }^{8}$ De formatione et reformatione ordinis Fratrum Praedicatorum is an intricate mosaic of quotations taken from the works of Aristotle, the Fathers of the Church, and some more recent theologians like Bernard of Clairvaux or Henry of Ghent. A quick count gives a result

the research in the article: Henryk Bitterfeld z Brzegu, "Przeglad Tomistyczny", 4, 1988, p. 179-190. A summary of the literature on Bitterfeld was done by Pavel Černuška in the introduction to the edition of Bitterfeld's texts on the Eucharist: Jindřich z Bitterfeldu, Eucharistické texty, ed. P. Černuška, Brno 2006, p. 52-72. Recently, a new hypothesis concerning Bitterfeld's biography appeared. Jakub Turek has argued that, after leaving Prague around 1396, Bitterfeld went to Wroclaw, where he was involved in the quarrel between mendicant friars and parish priests over hearing confession, and he wrote a new treatise De regimine hominis, see J. Turek, Andrzej Rusiniec i reforma obserwancka w polskiej prowincji dominikanów, Kwart. Hist., 127, 2019, p. 47.

${ }^{3}$ In my opinion, the treatise can be dated to the years 1388-1390. Vladimir Koudelka pinpoints precisely the date of composition of the treatise, without any reason I believe. He interprets the fragment of the treatise which mentions the flourishing Jewish state that fell due to the sins of its inhabitants, as an allusion to the pogrom of the Jews in Prague in the spring of 1389. Such an identification seems to me to be an overinterpretation, as it is much more likely that Bitterfeld meant the Roman conquest of Judea, cf. V.J. Koudelka, Heinrich Bitterfeld, p. 19-20.

${ }^{4}$ Observant Reform of the orders, including the Dominican Order, is enjoying growing scholarly interest, as seen in a number of publications, such as: A Companion to Observant Reform in the Late Middle Ages and Beyond, ed. J.D. Mixson, B. Roest, Brill's Companions to the Christian Tradition, Leiden 2018; A. Huijbers, Zealots for Souls. Dominican Narratives of Self-Understanding during Observant Reforms, c. 1388-1517, Berlin-Boston 2018; J.D. Mixson, Poverty's Proprietors: Ownership and Mortal Sin at the Origins of the Observant Movement, Leiden 2009; Reformbemühungen und Observanzbestrebungen im spätmittelalterlichen Ordenswesen, ed. K. Elm, Berlin 1989. A summary of research on the Observant movement to date can be found in the article by M. Lodone, Riforme e osservanze tra XIV e XVI secolo, "Mélanges de l'École française de Rome", 130/2, 2018, p. 267-287.

5 “[...] inventis quampluribus fratribus, qui ferventer desiderant reduci ad primam formam observantiae regularis, per beatissimum patrem nostrum Dominicum inchoatam, et per Sanctos sucessores eius postomodum consummatam [...] Decrevi [...], quod in qualibet provincia saepe dicti Ordinis sit ad minus unus conventus, in quo regularis observantia teneatur ad unguem iuxta nostrarum Constitutionum tenorem et formam. [...] Illos, quos invenerit, assignet in dicto conventu, in quo servetur ad unguem, ut dictum est, tenor Constitutionum nostrarum et observantia regularis, sicut in ipsis Constitutionibus est expressa [...]". It is with these words that Raymond of Capua, Master General of the Dominican Order, outlined his project of reform in the initiating decree of 1390, Beati Raymundi Capuani, XXIII magistri generalis Ordinis Praedicatorum Opuscula et litterae, ed. H.-M. Cormier, Romae 1895, p. 54-55. The central role of Order's law in the Observant Reform is also confirmed by Bitterfeld. He claims for example: "Quapropter ob [scripsi ad sensum] emendacionem tocius nostre conuersacionis satis necessarium uidetur materiam nostre Constitucionis ponderare, ut appareat singularius quantum a rectis limitibus de uia nos abduxerant, si uiam rectam mensurare uelimus", Bitterfeld, Tractatus, fol. 295v. All quotations from Bitterfeld's treatise come from the critical edition of the text I'm working on, which will appear in print in 2022. In the footnotes, I provide folio numbers in the manuscript of the University Library in Eichstätt (Ms. Eichstätt, Staatsbibliothek, 709, fols 267r-299r; quoted hereafter as: $\mathrm{ms}$ E).

${ }^{6}$ Acta Capitulorum Generalium, vol. 2: 1304-1378, ed. B.M. Reichert, Monumenta Ordinis Preadicatorum Historica 4, Romae 1899, p. 38.

7 On this subject, see for example: T. Gałuszka, Mikołaja Duthorowa "error condemnatus ab ecclesia". Dominikanie polscy wobec herezji i nowych nurtów pobożności w pierwszej połowie XIV w., Kwart. Hist., 121, 2014, p. 73-106.

${ }^{8}$ So far a treatise De contemplatione et vita activa (Henricus Bitterfeld de Brega OP, Tractatus de contemplatione et vita activa, ed. B. Mazur, W. Seńko, R. Tatrzyński, Warszawa 2003) and writings about the Eucharist (Jindřich z Bitterfeldu, Eucharistické texty) as well as some single sermons of Henry Bitterfeld have been published. 
of around two hundred quotations indicated by Henry himself (making up around sixty pages of the printed edition). The most popular sources are Thomas Aquinas (38 citations), Gregory the Great (39), Bernard of Clairvaux (37), Augustine (32), John Chrysostom (27), and Jerome (20). However, such a cursory review of auctoritates based on what the author himself indicates as a quotation can be very misleading. As all editors of medieval texts know, sometimes their authors provide erroneous or only very general identification of references. Sometimes they do not even mention that they are quoting somebody else and it is up to the erudition and keen eyes of the editor to notice that the author is not using his own words. Moreover, alongside very exact citations there are paraphrases, summaries, abbreviations etc.

One problem with identifying sources has its origins in the medieval understanding of authorship. According to St Bonaventure, there were four ways of creating a text in the Middle Ages, differing in the degree of use of other authors' texts: copying, compiling, commenting, and writing. ${ }^{9}$ Bonaventure gives an exact definition of each of those methods. Unfortunately, when confronted with medieval sources, this clear distinction fails. We know that even if the scribe had an intention to create a faithful copy, it was impossible for him to avoid errors of different kinds or not to introduce smaller or more significant changes in the wording or grammatical structure, without changing the meaning of the text. Thus, he - consciously or unconsciously - was creating a new version of the text he copied. This presents an editor with the problem of how far those changes can go so that we are still able to talk about a copy that can be used in a critical edition and not about a different version of the text; it, however, does not affect the question of identifying sources and creating apparatus fontium. The problem arises when it comes to the commentary and the compilation. We can imagine a commentary in the form of a compilation, when the author chooses one text to be commented on and the others as a comment. We could say that, in this case, a distinction between commentary and compilation is a purely academic discussion. Still, we have to be aware that the way we define the edited text may influence our approach to an edition. We can accurately identify the commented text and not notice quotations hidden within the commentary. Similar problems can arise with the text thought to be "original" work of the medieval author. According to Bonaventure, it should be written by one person, who uses quotations taken from other texts only to confirm his own thought. Editing practice shows that sometimes medieval scholars wanted to be seen as the authors in the strict sense of the term while, in fact, they were able compilers. Not only did they skip information about the authorship of single quotations, but also they took over, among other things, a structure, sets of arguments or auctoritates from other texts. Moreover, all those - hidden quotations, a commentary in the form of compilation, re-usage of the structures of other texts, etc. - can be sometimes observed in the very same text. Only through careful reading, usually together with a process of preparing a critical edition, do we have a chance to discover the real nature of the text in question. This is the case with Henry Bitterfeld's treatise De formatione et reformatione Ordinis Fratrum Praedicatorum and its relationship with the writings of Thomas Aquinas.

As mentioned before, if we roughly count quotations from Aquinas's works indicated by Bitterfeld, we find around forty of them, which makes Aquinas one of Bitterfeld's favourite authorities. However,

\footnotetext{
9 "Quadruplex est modus faciendi librum. Aliquis enim scribit aliena, nihil addendo vel mutando; et iste mere dicitur scriptor. Aliquis scribit aliena addendo, sed non de suo; et iste esse compilator dicitur. Aliquis scribit et aliena et sua, sed aliena tamquam principalia, et sua tamquam annexa ad evidentiam; et iste dicitur commentator non autor. Aliquis scribit et sua et aliena, sed sua tamquam principalia, aliena tamquam annexa ad confirmationem, et talis debet dici auctor", S. Bonaventura, Commentaria in Quatuor Libros Sententiarum, Lib. 1, Proemium, q. IV, Sancti Bonaventurae opera omnia 1, Ad Claras Aquas 1882, p. 14. On Bonaventure's distinction see E. Potkowski, Pisarz i jego dzieło w społeczeństwie średniowiecznym, in: id., Książka i pismo w średniowieczu. Studia z dziejów kultury piśmiennej i komunikacji społecznej, Pułtusk 2006, p. 306-309. On the medieval theory of authorship and discussion of the state of research on the issue, see e.g. A. Minnis, "Nolens auctor sed compilator reputari": the Late-medieval Discourse of Compilation, in: La méthode critique au Moyen Age, ed. M. Chazan, G. Dahan, Bibliothèque d'histoire culturelle du Moyen Age 3, collection dir. par N. Bériou et F. Morenzoni, Brepols 2006, p. 47-63.
} 
it is not the number of quotations but how Bitterfeld uses Aquinas's texts that makes the relationship between De formatione and the corpus of Aquinas's writings so exceptional. In Bitterfeld's work, St Thomas's texts do not only play the role of auctoritates, which can be put in a quotation mark with a corresponding footnote. Bitterfeld uses the corpus of Aquinas's texts, especially his treatise on religious life from Secunda Secundae of Summa Theologiae (q. 186-189) - they make up half of all the quotations taken from Aquinas's works - as a bilding material for his work.

The text of the treatise has a very complicated structure. It is divided into four major parts preceded by a prologue. Each of them is subject to multi-level divisions (up to eight levels of subdivisions). ${ }^{10}$ As a result, the text breaks down into dozens of particles. Only when we look closely at each of them, can we see the whole spectrum of methods used to combine Aquinas's works with the text of Bitterfeld. In some particles, Bitterfeld follows both the structure and the content of Aquinas's text, but supplements his reasoning with fragments of other works of the same author (1); sometimes he comments on a selected passage of Aquinas's work using Aquinas's writings to do so as well (2); and sometimes he treats the corpus of Aquinas's texts just as a useful source of auctoritates (3).

\section{Structure and content}

Sometimes Bitterfeld follows the structure of Aquinas's reasoning, extending some of its elements beyond Aquinas's words. This is the case with the opening part of the treatise, concerning chastity. In the opening section, Bitterfeld wants to prove that chastity is more directly subordinated to the perfect love of God than poverty. To explain this statement, he recalls two sets of arguments and several authorities. Aquinas is not mentioned among them. However, if we look closer, it is clear that Aquinas is nearly the only one.

Bitterfeld starts with a statement of Augustine (interestingly without naming the author): tanto magis inhaeretur Deo, quanto minus diligitur proprium, followed by a short explanation. Both - quotation and its exegesis - are taken from the eighth chapter of Aquinas's De perfectione spiritualis vitae. Then he presents two main arguments on behalf of the superiority of chastity over poverty. Both of them are taken directly from the corpus of the fourth article of question 186 of Secunda Secundae. Bitterfeld uses both the structure of Aquinas's argumentation and auctoritates collected by Aquinas (Aristotle, Augustine and Gregory the Great). However, this part of Bitterfeld's treatise is not just a copy of a part of the Summa. Bitterfeld extends Aquinas's arguments using for this purpose words of Aquinas himself, taken from De perfectione spiritualis vitae:

${ }^{10}$ For example, the second part of the first major part of the treatise (De religionum racionabili institucione) containing an explanation of the place of three religious vows in the regular life (Quomodo tria consilia predicta caritati deseruiunt et religioni et post hoc quomodo alia subordinantur istis) is subsequently divided into three parts (de paupertate, de castitate, de obedientia). Each of them breaks down into smaller parts. Subdivisions in the first part of the first part devoted to poverty go as follows:

I.2.1. de paupertate

I.2.1.1. votum paupertatis

I.2.1.1.1. deservit caritati quia disponit animam ad celestium desiderium et contemplacionem diuinorum

I.2.1.1.2. ualet ad humilitatem inducendam et conseruandam, que principaliter ad exercicium pertinet caritatis et eciam seruorum Dei

I.2.1.1.3. ualet ad feruorem caritatis frequenciorem, que nascitur ex predictis

I.2.1.1.4. ualet ad securitatem consciencie, que ex puritate uite est et fuga uiciorum

I.2.1.1.4.1. superbia

I.2.1.1.4.2. luxuria

I.2.1.1.4.3. gula

I.2.1.1.4.5. ira

I.2.1.1.4.6. avaritia

I.2.1.1.4.5. invidia

I.2.1.1.4.6. accidia 


\begin{tabular}{|c|c|}
\hline $\begin{array}{c}\text { Henricus Bitterfeld, } \\
\text { De formatione et reformatione } \\
\text { Ordinis Fratrum Praedicatorum } \\
\text { ms E, f. } 279 \mathrm{r}\end{array}$ & Thomas Aquinas $^{11}$ \\
\hline $\begin{array}{l}\text { Nunc sequitur de castitate, que inmediacius caritati et } \\
\text { perfeccioni religionis deseruit, quam paupertas. Quia, ut } \\
\text { supra habitum est, [1] tanto magis inheretur Deo, quanto } \\
\text { magis contempnitur proprium. Sed quia delectacio carnis } \\
\text { est nobis magis proprium ex parte corporis, quam temporales } \\
\text { diuicie, ideo renunciacio magis facit Deo inherere, quod est } \\
\text { effectus caritatis. Ibi exteriora relinquentur impedimenta } \\
\text { remota et aliena, hic propria et proxima et magis incentiua. } \\
\text { Et ut lucidius pateat, duplici racione inducetur. }\end{array}$ & $\begin{array}{l}\text { [De perfectione, cap. 8.] Ut autem secundam perfectionis viam } \\
\text { convenientius ostendamus, accipiendum est verbum Augustini, } \\
\text { qui dicit in } 12 \text { de Trin.: [1] tanto magis inhaeretur Deo, } \\
\text { quanto minus diligitur proprium. Secundum igitur ordinem } \\
\text { propriorum bonorum quae homo propter Deum contemnit, est } \\
\text { attendendus ordo eorum quibus ad perfectam Dei inhaesionem } \\
\text { pervenitur. Prius enim relinquenda occurrunt quae minus } \\
\text { nobis coniuncta existunt: unde in primo loco occurrit ad } \\
\text { perfectionem tendentibus exteriora bona relinquere, quae } \\
\text { a nostra natura sunt separata. Post haec vero relinquenda } \\
\text { occurrunt ea quae nobis naturae communione et affinitatis } \\
\text { cuiusque necessitate coniunguntur. }\end{array}$ \\
\hline $\begin{array}{l}\text { Prima est quia continencia deseruit feruori caritatis in } \\
\text { quantum a delectacione recedit, [2] ex cuius frequenti } \\
\text { experiencia augetur concupiscencia. Quod Philosophus } \\
\text { uult } 3^{\circ} \text { Ethicorum. Concupiscencia autem aliquando opponitur } \\
\text { caritati, secundum Glosam ad Romanos VII vel ad minus } \\
\text { tollit feruorem. [3] Et inde est quod usus uenerorum trahit } \\
\text { animam ab illa perfecta intencione tendendi in Deum. Vnde } \\
\text { Augustinus libro primo Soliloquiorum dicit: Nichil esse } \\
\text { sencio, quod magis ex arce deiciat animum uirilem quam } \\
\text { blandimenta femine corporumque ille contactus sine quo } \\
\text { uxor haberi non potest. Quanto enim operacio sensualis magis } \\
\text { intenditur tanto inferiorum uirium operacio magis remittitur, } \\
\text { propter quod feruor caritatis carnali delectacione prepeditur. } \\
\text { Propter hoc sacerdotes se pro sacrificio disponentes ab actu } \\
\text { coniugali in Veteri Lege debuerunt esse lib<e }>\text { ri et nunc } \\
\text { Ecclesie sancte constitucio perpetuam obseruanciam leuitatis } \\
\text { imponit. Nazarei eciam sic santificati mundicia uota sua sic Deo } \\
\text { commendabant, Spiritus Sanctus ora prophetarum tangebat. } \\
\text { Insuper et Apostolus dicit abstinendum esse ad tempus propter } \\
\text { oracionem, I Cor. VII [, 5]. Quod autem in omnibus illis nisi } \\
\text { ut feruor deuocionis magis excitaretur castitas singulis hiis } \\
\text { prestitit ydoneitatem. }\end{array}$ & $\begin{array}{l}\text { II }^{\text {a-II }}{ }^{\text {ae }} \text {, q. } 186 \text { a. } 4 \text { co. Respondeo dicendum quod ad statum } \\
\text { religionis requiritur subtractio eorum per quae homo impeditur } \\
\text { ne totaliter feratur ad Dei servitium. Usus autem carnalis } \\
\text { copulae retrahit animum ne totaliter feratur in Dei servitium, } \\
\text { dupliciter. Uno modo, propter vehementiam delectationis, } \\
\text { [2] ex cuius frequenti experientia augetur concupiscentia, } \\
\text { ut etiam philosophus dicit, in III Ethic. [3] Et inde est } \\
\text { quod usus venereorum retrahit animam ab illa perfecta } \\
\text { intentione tendendi in Deum. Et hoc est quod Augustinus } \\
\text { dicit, in I Soliloq., nihil esse sentio quod magis ex arce } \\
\text { deiiciat animum virilem quam blandimenta feminae, } \\
\text { corporumque ille contactus sine quo uxor haberi non potest. }\end{array}$ \\
\hline $\begin{array}{l}\text { [4] Secunda racio, quia castitas aufert sollicitudinem } \\
\text { negociorum temporalium, quam homines habere } \\
\text { consueuerunt de gubernacione uxorum et filiorum. Vnde } \\
\text { Apostolus dicit: Qui sine uxore est, sollicitus est, que } \\
\text { Domini sunt, qui autem cum uxore est, sollicitus est, que } \\
\text { sunt mundi et quomodo placeat uxori [cf. } 1 \text { Cor. } 7,32-33 \text { ]. }\end{array}$ & $\begin{array}{l}\text { [4] Alio modo, propter sollicitudinem quam ingerit homini } \\
\text { de gubernatione uxoris et filiorum, et rerum temporalium } \\
\text { quae ad eorum sustentationem sufficiant. Unde apostolus } \\
\text { dicit quod qui sine uxore est, sollicitus est quae sunt domini, } \\
\text { quomodo placeat Deo, qui autem cum uxore est, sollicitus } \\
\text { est quae sunt mundi, quomodo placeat uxori. } \\
\text { Et ideo continentia perpetua requiritur ad perfectionem } \\
\text { religionis, sicut et voluntaria paupertas. Unde sicut damnatus } \\
\text { est Vigilantius, qui adaequavit divitias paupertati; ita damnatus } \\
\text { est Iovinianus, qui adaequavit matrimonium virginitati. }\end{array}$ \\
\hline $\begin{array}{l}\text { [5a] Et hoc est uerbum Domini Luc. VIII }[14,26] \text { : Si quis } \\
\text { uenit ad me et non odit patrem suum et matrem, uxorem et } \\
\text { filios, non potest meus esse discipulus. Quare autem dicit tales } \\
\text { odio habendos, cum inimicos diligere debeamus, [5b] nisi } \\
\text { quod in eis diligamus, quod sunt et odio habemus, quod in } \\
\text { Dei itinere nobis obsistunt, ut Gregorius dicit. Hoc autem }\end{array}$ & $\begin{array}{l}\text { [De perfectione, cap. } 8 \text { ] [5a] Unde Dominus dicit, Luc. } \\
\text { XIV, 26: si quis venerit ad me, et non odit patrem suum } \\
\text { et matrem et uxorem et filios et fratres et sorores [...] } \\
\text { non potest meus esse discipulus. Sed percunctare libet, ut } \\
\text { Gregorius dicit, quomodo parentes et carnales amicos } \\
\text { praecipimur odisse, qui iubemur et inimicos diligere. Sed si }\end{array}$ \\
\hline
\end{tabular}

${ }^{11}$ All quotations from Thomas Aquinas's works are taken from the online edition Corpus Thomisticum, <http://www. corpusthomisticum.org/> [access: 26.07.2019]. 


\begin{tabular}{|c|c|}
\hline $\begin{array}{c}\text { Henricus Bitterfeld, } \\
\text { De formatione et reformatione } \\
\text { Ordinis Fratrum Praedicatorum } \\
\text { ms E, f. } 279 \mathrm{r}\end{array}$ & Thomas Aquinas \\
\hline $\begin{array}{l}\text { frequenter contingit. Adam enim quia Euam contristari noluit, } \\
\text { peccauit; Iudas, quia filiis prouidere uoluit, aliena rapuit et } \\
\text { magistrum uendidit. Denique multi parentes thesaurizant filiis, } \\
\text { ne deficiant cum usuris. Deseruit ergo castitas tam iusticie, } \\
\text { quam contemplacioni, in quantum animus minus sollicitudine } \\
\text { distrahitur et passione minus inpeditus, diuinis feruencius } \\
\text { poterit occupari. Vnde Commentator } 9 \text { Physicorum dicit, } \\
\text { quod castitas sapiencie studio confert, quia anima per hoc } \\
\text { magis a passione quiescit. Hoc enim Plato fecisse inuenitur } \\
\text { pro ueritatis contemplacione acquirende, ut Augustinus dicit } \\
\text { De uera religione. }\end{array}$ & $\begin{array}{l}\text { vim praecepti perpendimus, utrumque agere per discretionem } \\
\text { valemus [...] quasi enim per odium diligitur qui carnaliter } \\
\text { sapiens dum prava nobis ingerit, non auditur. Sic enim exhibere } \\
\text { proximis nostris odii discretionem debemus: [5b] ut in eis et } \\
\text { diligamus quod sunt, et habeamus odio quod in Dei nobis } \\
\text { itinere obsistunt. Quisquis enim iam aeterna concupiscit, } \\
\text { in eam quam aggreditur causam Dei, extra patrem, extra } \\
\text { matrem, extra uxorem, extra filios, extra cognatos, extra } \\
\text { semetipsum fieri debet; ut eo verius cognoscat Deum, quo } \\
\text { in eius causa neminem cognoscit. Manifestum namque est } \\
\text { quod carnales affectus intentionem mentis diverberant, eiusque } \\
\text { aciem obscurant. }\end{array}$ \\
\hline
\end{tabular}

\section{Commentary on and with Aquinas}

Sometimes the words of Aquinas and the way they are quoted in the text of Bitterfeld resemble a way in which the Bible is usually used. For example, at the very beginning of his treatise, Bitterfeld concludes his reflections on different meanings of the word "religio" (also based, by the way, on Aquinas's works) compared with a sentence taken from the third article of question 187: status religionis est exercicium siue disciplina, quo quis exercetur ad perfeccionem caritatis. This single sentence becomes the basis for drawing five conclusions.

\begin{tabular}{|c|c|}
\hline $\begin{array}{l}\text { Henricus Bitterfeld, } \\
\text { De formatione et reformatione } \\
\text { Ordinis Fratrum Praedicatorum } \\
\text { ms E, f. } 269 \mathrm{v}-270 \mathrm{r}\end{array}$ & $\begin{array}{l}\text { Thomas Aquinas } \\
\text { Summa theologiae }\end{array}$ \\
\hline $\begin{array}{l}{[\ldots] \text { Ideo dicit sanctus Thomas, quod status religionis est }} \\
\text { exercicium siue disciplina, quo quis exercetur ad perfeccionem } \\
\text { caritatis. }\end{array}$ & $\begin{array}{l}\mathrm{II}^{\mathrm{a}}-\mathrm{II}{ }^{\mathrm{ae}} \text {, q. } 186 \text { a. } 2 \text { co. [utrum religiosi teneantur ad omnia } \\
\text { Consilia] [...] Dictum est autem quod ipsa perfectio caritatis } \\
\text { est finis status religionis, status autem religionis est quaedam } \\
\text { disciplina vel exercitium ad perfectionem perveniendi. }\end{array}$ \\
\hline $\begin{array}{l}\text { [1] Ex quo apparet, quod religio, ut hic sumitur est in consiliis, } \\
\text { quia illa ordinantur ad perfeccionem caritatis, ut apparebit } \\
\text { in sequentibus, quoad tria predicta. }\end{array}$ & $\begin{array}{l}\mathrm{II}^{\mathrm{a}}-\mathrm{II}^{\mathrm{a} e} \text {, q. } 187 \text { a. } 2 \text { co. [utrum liceat eis de negotiis saecularibus } \\
\text { se intromittere] Respondeo dicendum quod, sicut supra dictum } \\
\text { est, status religionis est ordinatus ad perfectionem caritatis } \\
\text { consequendam. }[\ldots]\end{array}$ \\
\hline $\begin{array}{l}\text { [2] Secundo infertur, quod religio presupponit caritatem, quia } \\
\text { non dicitur, quod religio disponit ad caritatem sed ad eius } \\
\text { perfeccionem, que habetur ex feruore et intensione. Sed dicit } \\
\text { sanctus Thomas, quod consilia ordinantur ad remouendum } \\
\text { impedimenta actus perfecte caritatis. }[\ldots]\end{array}$ & $\begin{array}{l}\mathrm{II}^{\mathrm{a}}-\mathrm{II}^{\mathrm{ae}} \text {, q. } 189 \text { a. } 1 \text { co. [utrum illi qui non sunt exercitati } \\
\text { in observantia praeceptorum, debeant religionem ingredi] } \\
\text { Respondeo dicendum quod, sicut ex supra dictis patet, status } \\
\text { religionis est quoddam spirituale exercitium ad consequendum } \\
\text { perfectionem caritatis, quod quidem fit inquantum per } \\
\text { religionis observantias auferuntur impedimenta perfectae } \\
\text { caritatis. [...] }\end{array}$ \\
\hline $\begin{array}{l}\text { [3] Tercio principaliter infertur, quod religio presupponit totam } \\
\text { uitam moralem et euitacionem omnium peccatorum mortalium, } \\
\text { quia et hoc requiritur ad caritatem, quam presupponit, ut } \\
\text { dictum est. [...] }\end{array}$ & \\
\hline $\begin{array}{l}\text { [4] Quartum est, quod sicut consilia ordinantur ad } \\
\text { perfeccionem caritatis, scilicet tria principalia, sic et } \\
\text { Constituciones et Regule et patrum ammoniciones ordinantur } \\
\text { ad conseruacionem et exercicium consiliorum. [...] }\end{array}$ & $\begin{array}{l}\mathrm{II}^{\mathrm{a}}-\mathrm{II}^{\mathrm{ae}} \text {, q. } 189 \text { a. } 1 \text { ad } 5 \text { Ad quintum dicendum quod } \\
\text { praeceptorum quaedam sunt principalia, quae sunt fines et } \\
\text { praeceptorum et consiliorum, scilicet praecepta caritatis. Ad } \\
\text { quae consilia ordinantur, non ita quod sine consiliis servari } \\
\text { non possint, sed ut per consilia perfectius observentur. [...] }\end{array}$ \\
\hline
\end{tabular}




\begin{tabular}{|c|l|}
\hline \multicolumn{1}{|c|}{$\begin{array}{c}\text { Henricus Bitterfeld, } \\
\text { De formatione et reformatione } \\
\text { Ordinis Fratrum Praedicatorum } \\
\text { ms E, f. 269v-270r }\end{array}$} & \multicolumn{1}{c|}{$\begin{array}{c}\text { Thomas Aquinas } \\
\text { Summa theologiae }\end{array}$} \\
\hline $\begin{array}{l}\text { [5] Quintum est, quod [1] omnes religiones conueniunt in } \\
\text { tribus uotis sed differunt in hoc, quod aliqua religio habet } \\
\text { meliora et faciliora et expediciora media siue adminicula ad } \\
\text { perfeccionem caritatis ordinata, una quam alia. In hoc una } \\
\text { enim dicitur superior ad aliam. }\end{array}$ & $\begin{array}{l}\text { II }{ }^{\text {a }-\mathrm{II}}{ }^{\mathrm{ae}} \text {, q. } 188 \text { a. 6 co. [utrum religio quae ordinatur ad } \\
\text { vetivam] Respondeo dicendum quod, sicut supra dictum } \\
\text { est, [1] differentia unius religionis ad aliam principaliter } \\
\text { quidem attenditur ex parte finis, secundario autem ex parte } \\
\text { exercitii. Et quia non potest aliquid dici altero potius nisi } \\
\text { secundum id in quo ab eo differt, ideo excellentia unius } \\
\text { religionis super aliam principaliter quidem attenditur } \\
\text { secundum religionis finem, secundario autem secundum } \\
\text { exercitium. [...] }\end{array}$ \\
\hline
\end{tabular}

It is a typical rhetorical move used by preachers when, on the basis of the so-called verba thematis, they introduce a division organising the structure of the whole sermon. Three of those conclusions are supported with more or less extensive quotations taken from different parts of the treatise mentioned above on religious life from Secunda Secundae of the Summa theologiae. We know this type of exegesis from commentaries on the Bible where scholars are explaining one passage of the Bible with another.

At the same time, the fragment quoted above is not only an example of a comment on Aquinas's text but also a compilation made of fragments drawn from different parts of his work. Especially interesting is the fifth conclusion devoted to the issue of differences between religious orders. The whole fragment shows a deep dependence on the sixth article of q. 186 being an answer to the question: Utrum religio quae ordinatur ad vitam contemplativam, sit potior ea quae ordinatur ad vitam activam, even though Bitterfeld does not indicate Aquinas as his auctoritas here.

\section{Henricus Bitterfeld, De formatione et reformatione Ordinis Fratrum Praedicatorum ms E, f. 270r-v}

[fragment with a commentary on: Status religionis est exercicium siue disciplina, quo quis exercetur ad perfeccionem caritatis]

[1] Omnes religiones conueniunt in tribus uotis sed differunt in hoc, quod aliqua religio habet meliora et faciliora et expediciora media siue adminicula ad perfeccionem caritatis ordinata, una quam alia. In hoc una enim dicitur superior ad aliam.

[3] Vna enim ordinatur ad uitam actiuam et ista est sub illa, que ordinatur ad uitam contemplatiuam et actiuam. Exemplum: sicut militaris religio, ut Cruciferorum et Iohannitarum ordinatur ad Ecclesie defensionem contra infideles et quia propter defensionem corporis, non instruccionem anime uidetur instituta, ideo est actiua tantum et est inferior aliis.

Aliqua uero ordinatur ad contemplatiuam, sicut est uita monastica ruralis, de qua dicit Ieronimus Ad Paulinum, quod sancta rusticitas non prodest nisi sibi.

Et illa est sub tercia, [2] que ordinatur principaliter ad contemplatiuam sed ex contemplacione descendunt tales ad actiuam, sicut est religio, que ordinatur ad predicandum. Ideo subiungit ibi Ieronimus, quod melior est docta iusticia, quia qui docti fuerint, fulgebunt sicut splendor firmamenti et qui ad iusticiam erudiunt multos quasi stelle in perpetuas eternitates, talis inmediate est sub statu episcoporum, qui excedit omnem

\section{Thomas Aquinas,}

Summa theologiae, II $^{\text {a }}-\mathrm{II}^{\text {ae }}$, q. 188 a. 6 co. [Utrum religio quae ordinatur ad vitam contemplativam, sit potior ea quae ordinatur ad vitam activam]

Respondeo dicendum quod, sicut supra dictum est, [1] differentia unius religionis ad aliam principaliter quidem attenditur ex parte finis, secundario autem ex parte exercitii. Et quia non potest aliquid dici altero potius nisi secundum id in quo ab eo differt, ideo excellentia unius religionis super aliam principaliter quidem attenditur secundum religionis finem, secundario autem secundum exercitium. Diversimode tamen secundum utrumque comparatio attenditur, nam comparatio quae est secundum finem est absoluta, eo quod finis propter se quaeritur; comparatio autem quae est secundum exercitium, est respectiva, quia exercitium non quaeritur propter se, sed propter finem. Et ideo illa religio alteri praefertur quae ordinatur ad finem absolute potiorem, vel quia est maius bonum; vel quia ad plura bona ordinatur. Si vero sit finis idem, secundario attenditur praeeminentia religionis, non secundum quantitatem exercitii, sed secundum proportionem eius ad finem intentum. Unde et in collationibus patrum introducitur sententia beati Antonii, qui praetulit discretionem, per quam aliquis omnia moderatur, et ieiuniis et vigiliis et omnibus huiusmodi observantiis. Sic ergo dicendum est quod opus vitae activae est duplex. [2] Unum quidem quod 
religionem. Si tamen predicacio esset impedimentum contemplacionis, sic quod ex contemplacione non procederet sed magis animam distraharet, ut multis contingit ex cupiditate predicacionis, tunc non esset uerum, secundum quod dicit Heinricus de Gandauo, Quotlibetum 12, q. 29: Non igitur est melior religio dicenda, que habet plura ieiunia aut austeriora precepta, sed que habet expediciora media ad exercicium caritatis ordinata et in perfeccionem caritatis ducencia, quantum ad se et ad alios. Dico autem expediciora simpliciter, que inmediacius promouent ad actum feruentem caritatis et contemplacionis gradum superiorem. ex plenitudine contemplationis derivatur, sicut doctrina et praedicatio. Unde et Gregorius dicit, in V Homil. super Ezech., quod de perfectis viris post contemplationem suam redeuntibus dicitur, memoriam suavitatis tuae eructabunt. Et hoc praefertur simplici contemplationi. Sicut enim maius est illuminare quam lucere solum, ita maius est contemplata aliis tradere quam solum contemplari. [3] Aliud autem est opus activae vitae quod totaliter consistit in occupatione exteriori, sicut eleemosynas dare, hospites recipere, et alia huiusmodi. Quae sunt minora operibus contemplationis, nisi forte in casu necessitatis, ut ex supra dictis patet. Sic ergo summum gradum in religionibus tenent quae ordinantur ad docendum et praedicandum. Quae et propinquissimae sunt perfectioni episcoporum, sicut et in aliis rebus fines primorum coniunguntur principiis secundorum, ut Dionysius dicit, VII cap. de Div. Nom. Secundum autem gradum tenent illae quae ordinantur ad contemplationem. Tertius est earum quae occupantur circa exteriores actiones. In singulis autem horum graduum potest attendi praeeminentia secundum quod una religio ordinatur ad altiorem actum in eodem genere, sicut inter opera activae, potius est redimere captivos quam recipere hospites; et in operibus contemplativae, potior est oratio quam lectio. Potest etiam attendi praeeminentia, si una earum ad plura horum ordinetur quam alia, vel si convenientiora statuta habeat ad finem propositum consequendum.

\section{Florilegium}

Sometimes Bitterfeld uses Aquinas only as a source of quotations. However, he does this in a quite sophisticated way. For example, when he is trying to prove that mendicant orders are the perfect ones because begging gives friars occasion to exercise themselves in humility, he recalls fifth article of question 187 of Secunda Secundae. Aquinas gives there an answer as to whether those in religious orders should beg. A quotation from Aquinas is followed by ones from Canon Law and St Jerome, and examples from St Alexius of Rome and Arsenius the Great. When we go to the quoted article, it turns out that not only the opening sentence but the whole quotation is taken from Aquinas's work. Apparently, Bitterfeld was interested not in the argumentation with which his saintly brother supported a different (but not contradictory) thesis, but in a set of authorities used by Aquinas he found useful.

\begin{tabular}{|c|c|}
\hline $\begin{array}{c}\text { Henricus Bitterfeld, De formatione et reformatione } \\
\text { Ordinis Fratrum Praedicatorum, } \\
\text { ms E, f. } 273 \mathrm{v}\end{array}$ & $\begin{array}{c}\text { Thomas Aquinas, Summa theologiae, } \\
\text { II }^{\text {a }}-\mathbf{I I}^{\mathrm{ae}}, \mathbf{q} .187 \text { a. } 5 \text { co. } \\
\text { [Utrum liceat religiosis mendicare] }\end{array}$ \\
\hline $\begin{array}{l}\text { [...] religio mendicancium aliis est preferenda, quia conseruat } \\
\text { paupertatem magis humilitati deservientem. Vnde Innocencius } \\
\text { libro De uilitate condicionis humane dicit: "O miserabilis } \\
\text { condicio mendicitatis! Si petit, pudore confunditur, si non, } \\
\text { egestate consumitur sed ut mendicet, necessitate compellitur". } \\
\text { Talis autem pudor humilitatem generat et necessitas paupertatem } \\
\text { conservat. } \\
\text { De primo dicit sanctus Thomas Secunda Secunde q. 187, } \\
\text { articulo } 5^{\text {to }} \text {, [1] quod mendicitas est eficacissima medicina } \\
\text { contra superbiam, propter assumpcionem abieccionis. [2] De } \\
\text { pe(nitencia) dist. } 3^{\text {a }} \text { capitulo: Si quis se uilioribus officiis subdat } \\
\text { et ministeriis indignioribus tradat, ita namque arrogancie } \\
\text { et humane glorie uicium curare poterit. Et Ieronimus }\end{array}$ & $\begin{array}{l}\text { Respondeo dicendum quod circa mendicitatem duo possunt } \\
\text { considerari. Unum quidem ex parte actus ipsius mendicationis, } \\
\text { quae habet quandam abiectionem sibi coniunctam, illi enim } \\
\text { videntur abiectissimi inter homines esse qui non solum sunt } \\
\text { pauperes, sed in tantum sunt egentes quod necesse habent ab } \\
\text { aliis victum acquirere. Et secundum hoc, causa humilitatis } \\
\text { aliqui laudabiliter mendicant, sicut et alia assumunt quae ad } \\
\text { abiectionem quandam pertinent, quasi [1] efficacissimam } \\
\text { medicinam contra superbiam, quam vel in seipsis, vel etiam } \\
\text { in aliis per exemplum exstinguere volunt. Sicut enim infirmitas } \\
\text { quae est ex superexcessu caloris, efficacissime sanatur per ea } \\
\text { quae in frigiditate excedunt; ita etiam pronitas ad superbiam } \\
\text { efficacissime curatur per ea quae multum abiecta videntur. Et } \\
\text { ideo dicitur in decretis, [2] de Poenit., dist. II, cap. si quis }\end{array}$ \\
\hline
\end{tabular}


in epistula ad Occeannum commendat Fabiolam, de hoc quod optabat, ut suis diviciis pariter effusis pro Christo, stipes acciperet. [3] Exemplum de beato Alexio et Arsenio. Tamen nota cupiditatis excludi debet. Magis ergo factum est fratres hostiatim mendicare, quam monachos possesionibus ex communitate habundare, quas deridet Gregorius in Moralibus dicens: "Pauperes esse volunt sine defectu et humiles sine despectu. Paupertatem diligentes ut nullam inopiam paciantur". semel, exercitia humilitatis sunt, si quis se vilioribus officiis subdat, et ministeriis indignioribus tradat, ita namque arrogantiae et humanae gloriae vitium curari poterit. Unde Hieronymus, in epistola ad Oceanum, commendat Fabiolam de hoc quod optabat ut, suis divitiis pariter effusis pro Christo, stipes acciperet. [3] Quod etiam beatus Alexius perfecit, qui, omnibus suis propter Christum dimissis, gaudebat se etiam a servis suis eleemosynas accepisse. Et de beato Arsenio legitur, in vitis patrum, quod gratias egit de hoc quod, necessitate cogente, oportuit eum eleemosynam petere. [...]

Around 15 percent of all auctoritates in Bitterfeld's work are taken from the works of Aquinas.

\section{Conclusion}

The analyses presented above of excerpts from Bitterfeld's treatise show him as a diligent student of Aquinas's works. References to Aquinas's writings in De reformatione are not just rhetorical moves or obligatory elements which have to be used to make the text acceptable for the authorities of the Order. On the contrary, Bitterfeld's treatise shows that he knew Aquinas's teaching deeply and made it his own way of thinking. This faithful adherence to the doctrine of Aquinas seems to be a hallmark of Dominican observants. Also, the sermons of the father of Polish Dominican observants John Frankenstein are deeply dependent on Aquinas's works. ${ }^{12}$ Still, the question of the reception of Aquinas's text among Polish friars and preachers requires further in-depth study. ${ }^{13}$

The case of Bitterfeld's treatise and his use of Aquinas's works shows how complicated the question of authorship in the Middle Ages is and, in consequence, how vigilant the editor of medieval texts has to be. The very well-known and widely-discussed distinction taken from Bonaventure's Commentary on the Sentences of Peter Lombard, between auctor, commentator, compilator and scriptor is useful in the analysis of late-medieval texts, but it is not sufficient. It transpires that the De reformatione, like many other late-medieval works, is not just a simple text inlaid with quotations, in which aliquis scribit et sua et aliena, sed sua tamquam principalia, aliena tamquam annexa ad confirmationem, because at the same time it is a compilation and a commentary according to Bonaventure's distinction. Authoritative texts, like Aquinas's works in Bitterfeld's treatise, can play a role far beyond that attributed to authorities. Extended quotations are reworked, deconstructed, compiled and sometimes hidden deep in the structure of the text. Bitterfeld not only quotes Aquinas, he enters into dialogue with him, not to undermine his opinions, but to develop and explain them. This intertextual character of the text can be discovered and analysed only on the basis of a good critical edition. That is one of the reasons why an extensive programme of editorial works on medieval texts is essential for future research.

\section{Henry Bitterfeld, Thomas Aquinas and Identifying Sources in Late Medieval Texts. A Case Study of De formatione et reformatione Ordinis Fratrum Praedicatorum}

Summary: Research into the writings of Dominican Henry Bitterfeld of Brzeg revealed him to be an able compiler whose texts are a mosaic of quotations taken from the writings of ancient and medieval authors. This is also the case with his treatise De formatione et reformatione Ordinis Fratrum Praedicatorum, written for the Dominican Observant Reform movement gathering speed at the end of the $14^{\text {th }}$ century. Special attention is drawn to the numerous references to St Thomas Aquinas. In the context of Bitterfeld's treatise, these references are of particular importance, since by the regulation of the Dominicans General Chapter, St Thomas's doctrine was adopted as

\footnotetext{
12 See: A. Zajchowska, Między uniwersytetem a zakonem. Biografia i spuścizna pisarska dominikanina Jana z Ząbowic (zm. 1446), Warszawa 2013, p. 92-127.

13 On the place of Aquinas's thought among Polish Dominicans see: S. Swieżawski, Studium św. Tomasza u dominikanów w wieku XV, "Roczniki Filozoficzne", 17, 1969, nr 1, p. 21.
} 
obligatory for the whole Order. Bitterfeld, however, was a theoretician of the emerging Observance movement, propagating the return of the Dominicans to the faithful observance of the Order's regulations. In the light of the analysis of the treatise on the Order's reform, Henry Bitterfeld appears as a diligent and faithful pupil of St Thomas Aquinas, eager to give a special place to Aquinas's doctrine in his writings. Not only is Aquinas the most often quoted authority in Bitterfeld's treatise but also his texts - and especially the treatises on the monastic life Summa Theologiae Pars Secunda Secundae and De perfectione spiritualis vitae - make the building materials for Bitterfeld's own writings. The article presents an analysis of three methods in which Bitterfeld uses Aquinas's texts in his De reformatione. At times, Bitterfeld takes a fragment of St Thomas's writing and, following its structure and content, supplements it with pieces of other works and his own thoughts; sometimes he comments on a selected passage of Aquinas's work using other writings by Aquinas; and sometimes he treats the corpus of Aquinas's texts as a kind of forilegium - a collection of quotations. Bitterfeld's broad array of methods he used to compile a text with generous use of writings by other authors emphasises the methodological problems in research into medieval philosophical and theological texts resulting from the attitude towards the authorship in the Middle Ages.

Nota 0 autorze: Anna Zajchowska-Bołtromiuk, dr hab., prof. UKSW, mediewistka zatrudniona na Wydziale Nauk Historycznych UKSW. Absolwentka Uniwersytetu Jagiellońskiego, gdzie w 2009 r. uzyskała doktorat. W 2018 r. uzyskała habilitację na Uniwersytecie im. Adama Mickiewicza w Poznaniu. Jej zainteresowania badawcze koncentrują się na kulturze intelektualnej późnego średniowiecza, zwłaszcza zakonie dominikańskim. Jest autorką wielu prac poświęconych kaznodziejstwu mendykanckiemu i hagiografii.

Author: Anna Zajchowska-Bołtromiuk, dr hab., prof. UKSW, medievalist, Professor at the Faculty of History of the Cardinal Stefan Wyszynski University in Warsaw. She completed PhD studies at the Jagiellonian University and obtained her habilitation degree at the University of Adam Mickiewicz in Poznań. Her research interests focus on the intellectual culture of the Late Middle Ages, especially on the Dominican Order. She is the author of numerous works on mendicant preaching and hagiography.

Wydział Nauk Historycznych

Uniwersytetu Kardynała Stefana Wyszyńskiego w Warszawie

ul. Wóycickiego $1 / 3$ bud. 23

01-938 Warszawa

e-mail: aniazaj@gmail.com

\section{Bibliography}

Koudelka V.J., Heinrich Bitterfeld. Profesor an der Universität Prag, "Archivum Fratrum Praedicatorum”, 23, 1953, p. 5-65

Mill-Papuzińska M., Henryk Bitterfeld z Brzegu, "Przeglad Tomistyczny", 4, 1988, p. 179-190

Minnis A., "Nolens auctor sed compilator reputari": the Late-medieval Discourse of Compilation, in: La méthode critique au Moyen Age, ed. M. Chazan, G. Dahan, Brepols 2006, Bibliothèque d'histoire culturelle du Moyen Age, 3, p. 47-63

Potkowski E., Pisarz i jego dzieło w społeczeństwie średniowiecznym, in: id., Książka i pismo w średniowieczu. Studia z dziejów kultury piśmiennej i komunikacji społecznej, Pułtusk 2006, p. 290-311

Reformbemühungen und Observanzbestrebungen im spätmittelalterlichen Ordenswesen, ed. K. Elm, Berlin 1989

Swieżawski S., Studium św. Tomasza u dominikanów w wieku XV, "Roczniki Filozoficzne", 17, 1969, no. 1, p. 19-40

Turek J., Andrzej Rusiniec i reforma obserwancka w polskiej prowincji dominikanów, Kwart. Hist., 127, 2019, p. 375-397

Zajchowska A., „Quomodo cecidisti?” - kryzys w Zakonie Braci Kaznodziejów w średniowieczu w świetle traktatu Henryka Bitterfelda „De formatione et reformatione Ordinis Fratrum Praedicatorum”, “Czas Przeszły. Poznańskie Studia Historyczne", 4, 2017, p. 125-135

Zajchowska A., Formatio - deformatio - reformatio. O reformie obserwanckiej Zakonu Braci Kaznodziejów w świetle traktatu "De (de)formatione et reformatione" Henryka Bitterfelda, in: "Ecclesia semper reformanda". Kryzysy i reformy średniowiecznego Kościoła, ed. T. Gałuszka, T. Graff, G. Ryś, Kraków 2013, p. 507-514

Zajchowska A., Między uniwersytetem a zakonem. Biografia i spuścizna pisarska dominikanina Jana z Ząbkowic (zm. 1446), Warszawa 2013 